Reprod. Nutr. Dévelop., 1980, 20 (1 B), 311-321.

\title{
Influence de l'hyperinsulinémie sur les récepteurs d'insuline dans le foie du rat en croissance soumis à une restriction énergétique
}

\author{
par J. GRIZARD, M. ARNAL, R. PION \\ avec la collaboration technique de Françoise BARRE \\ Laboratoire d'Efude du Métabolisme Azoté, I.N.R.A. \\ Theix, Saint-Genès-Champanelle, 63110 Beaumont.
}

Summary. Effect of experimental hyperinsulinemia on insulin binding in liver plasma membranes from growing rats subjected to energetic restriction.

Two experiments (I and II) were carried out to study the interaction of insulin with amino acid metabolism in rats subjected to a restricted energy allowance (Grizard et al., 1975). 61 male rats, weighing $94 \mathrm{~g}$ at the beginning of the experiments, were divided into 4 groups and fed every 4 hrs. Group T was fed a balanced diet, and groups -E1, -E2 and -E3 were given a restricted energy allowance (table 1). The rats of group -E3 were injected with insulin at $11 \mathrm{a}$. m. every day, and those of group -E2 at that hour on the day they were killed. When the animals of a group had a mean body weight of $157 \mathrm{~g}$, they were killed between 2 p. m. and 6 p. m., i. e. between two meals. Plasma insulin and insulin binding to liver plasma membranes were recorded.

Reducing dietary energy intake resulted in a small decrease of postprandial plasma insulin and in a small increase of the plasma insulin clearance rate (table 2). It also augmented $\left({ }^{125} \mid\right.$ ) iodoinsulin binding to liver plasma membranes (fig. 1). This change could explain the increment of the liver protein synthesis observed in such animals (Arnal, Fauconneau and Pech, 1972).

Exogenous insulin induced a resistance to insulin effects (Grizard, Prugnaud and Pion, 1977), and caused a large increase of plasma insulin and a small decline in (125!) iodoinsulin binding to liver plasma membranes for 3 to $5 \mathrm{~h}$ after the injection (fig. 1). A large increment in insulin binding was then noted (fig. 1). Increased insulin binding and decreased plasma insulin might be correlated. The enhanced insulin binding could not explain the resistance to the effects of insulin.

La restriction énergétique entraîne une forte réduction de la croissance et de l'efficacité alimentaire apparente des protéines (Rérat et Desmoulin, 1970) ; celles-ci s'expliquent en partie par un ralentissement de l'anabolisme protéique musculaire associé à une augmentation de la synthèse de protéines hépatiques et de l'utilisation des acides aminés à des fins énergétiques (Arnal, Fauconneau et Pech, 1972). Le présent travail a pour but de voir dans quelle mesure les modifications du métabolisme azoté chez les animaux soumis à la restriction énergétique, sont la conséquence d'une dimi- 
nution des effets de l'insuline : augmentation du transporł des acides aminés dans le muscle, accroissement de la synthèse et ralentissement du catabolisme protéique dans le muscle ef le foie (Fulks, Li et Golberg, 1975 ; Bellemann ef al., 1977), ef diminution de la néoglucogenèse hépatique (Parilla, Jimenez et Ayuso-Parilla, 1976). II complète les travaux de Grizard et al. (1975) qui indiquent que l'insulinémie des rats soumis à la restriction énergétique n'est pas modifiée alors que le traitement par de fortes doses d'insuline exogène, accroît la rétention azotée.

C'est pourquoi nous avons étudié l'activité des récepteurs d'insuline dans les membranes plasmiques du foie de rats en croissance soumis à une restriction énergétique, en particulier les constantes cinétiques et les variations induites par l'hormone. En effet, l'interaction de l'insuline avec les récepteurs membranaires de cette hormone est un événement indispensable à l'action cellulaire de l'insuline (Freychet, 1976a).

\section{TABLEAU I}

Consommation, croissance ef efficacité alimentaire

\begin{tabular}{|c|c|c|c|c|c|}
\hline \multirow{2}{*}{$\frac{\text { Experience } \ldots \ldots \cdots \cdots \cdots}{\text { Lots } \ldots \ldots \ldots \ldots \ldots \ldots}$} & \multicolumn{2}{|c|}{1} & \multicolumn{2}{|c|}{ II } & \multirow{4}{*}{$\begin{array}{c}\text { Pourcentage } \\
\text { de } \\
\text { variation } \\
\text { moyen }\end{array}$} \\
\hline & $\mathrm{T}$ & $-\mathrm{E} 1$ & $-E 2$ & $-E 3$ & \\
\hline Animaux .............. & Témoins & \multicolumn{3}{|c|}{ Soumis à la restriction énergétique } & \\
\hline Traitement par l'insuline .. & - & - & 1 injection & $\begin{array}{l}\text { injections } \\
\text { journalières }\end{array}$ & \\
\hline Nombre d'animaux ...... & 15 & 15 & 15 & 16 & \\
\hline Durée de l'expérience (j) ... & 12 & 21 & 21 & 21 & \\
\hline $\begin{array}{l}\text { Poids moyen au moment du } \\
\text { sacrifice }(g) \ldots \ldots \ldots \ldots\end{array}$ & 156 & 162 & 156 & 155 & 5,3 \\
\hline $\begin{array}{c}\text { Matière sèche ingérée } \\
(\mathrm{g} / \mathrm{rat} / \mathrm{j})(\mathbf{4})\end{array}$ & $14,5_{123}$ & $10,3_{t}$ & $9,9 \mathrm{t}$ & $9,9_{\mathrm{t}}$ & \\
\hline $\begin{array}{r}\text { Energie ingérée }(\mathrm{kcal} / \mathrm{rat} / \mathrm{j}) \\
\left({ }^{4}\right) \ldots \ldots \ldots \ldots \ldots \ldots \ldots \ldots \\
\end{array}$ & $62,8_{123}$ & $44,6 \mathrm{t}$ & $42,8_{\mathrm{t}}$ & $42,99_{t}$ & 4,1 \\
\hline $\begin{array}{r}\mathrm{N} \times 6,25 \text { ingéré }(\mathrm{g} / \mathrm{rat} / \mathrm{j}) \\
\left({ }^{4}\right) \ldots \ldots \ldots \ldots \ldots \ldots \ldots \cdots\end{array}$ & $1,71_{123}$ & $2,02_{t}$ & $1,94_{t}$ & $1,94_{t}$ & \\
\hline $\begin{array}{l}\text { Croissance moyenne }(\mathrm{g} / \mathrm{j}) \\
\left({ }^{4}\right) \ldots \ldots \ldots \ldots \ldots \ldots \ldots \cdots\end{array}$ & $5,34_{123}$ & $3,47_{t_{23}}$ & $3,01_{t_{1}}$ & $3,07_{t_{1}}$ & 12,6 \\
\hline $\begin{array}{l}\text { Indice de consommation (g } \\
\text { de } M S \text { ingéré par g de } \\
\text { croît).................. }\end{array}$ & $2,74_{23}$ & $2,98_{23}$ & $3,34_{t_{1}}$ & $3,29_{t_{1}}$ & \\
\hline $\begin{array}{c}\text { CEP ( } g \text { de croît par } g \text { de } \\
\text { protéine ingérée) } \ldots \ldots \ldots \ldots\end{array}$ & $3,13_{123}$ & $1,72_{t_{23}}$ & $1,55_{t_{1}}$ & $1,58_{t_{1}}$ & 10,6 \\
\hline $\begin{array}{c}\text { CEE (g de croît par } 1000 \\
\text { kcal ingérées) ........... }\end{array}$ & $85,0_{123}$ & $77,8_{t_{23}}$ & $70,2_{t_{1}}$ & $71,5_{t_{1}}$ & \\
\hline
\end{tabular}

t, 1, 2, 3 : Moyenne significativement différente $(P \leqslant 0,050)$ des moyennes respectives obtenues pour les lots correspondants $T,-E 1,-E 2$ et $-E 3$.

( $\left.{ }^{1}\right)$ Mesurés pendant les 7 et les 13 jours qui précèdent le sacrifice respectivement pour le lot T et les lots -E. 


\section{Matériel et méthodes.}

Deux expériences ( $l$ et II) sont réalisées au moyen de 61 rats mâles de souche Sprague Dawley élevés dans les mêmes conditions que précédemment (Grizard et al., 1975 ; Grizard, Prugnaud et Pion, 1977). Les animaux sont sevrés vers $40 \mathrm{~g}$ et nourris soit d'un régime standard (expérience I), soit d'un régime standard puis du régime témoin (expérience II). Lorsque leur poids vif est voisin de $94 \mathrm{~g}$, les rats sont répartis en 4 lots : $T$ et $-E 1$ dans l'expérience I, et -E2 et -E3 dans l'expérience II (tabl. 1). Les rats du lot $T$ reçoivent le régime témoin tandis que ceux des lots - $E$ sont soumis à une restriction énergétique. La composition des régimes a été précédemment décrite (Grizard ef al., 1975), à l'exception de la supplémentation en L-thréonine qui est égale à 0,80 ef $1,33 \mathrm{~g}$ par $\mathrm{kg}$ de matière sèche, respectivement pour les régimes consommés par les animaux du lot témoin, et les animaux soumis à la restriction énergétique. Les rats reçoivent les aliments en 6 repas égaux par jour, distribués à 10,14,18, 22, 2 et $6 \mathrm{~h}$. Les rats du lot -E3 reçoivent des injections sous-cutanées d'insuline (insuline zinc mixte Novo lente, lot $721-8$ ), effectuées tous les jours à $11 \mathrm{~h}$; la dose diluée dans $0,5 \mathrm{ml}$ de soluté physiologique, est augmentée progressivement de 0,25 à $0,40 \mathrm{UI}$ par $100 \mathrm{~g}$ de poids vif, à la fin du traitement. Les rats du lot -E2 reçoivent une injection d'insuline seulement le jour du sacrifice $(0,22$ Ul par $100 \mathrm{~g}$ de poids vif) ; ils reçoivent des injections de soluté physiologique tous les autres jours de la période expérimentale. Lorsqu'ils ont atteint un poids moyen de $157 \mathrm{~g}$, les animaux de chaque loł sont sacrifiés le même jour, par égorgement sans anesthésie ; les sacrifices sont régulièrement répartis entre les repas de 14 et $18 \mathrm{~h}$. Le sang est recueilli sur héparine ; une partie de celui-ci est centrifugée pour la séparation du plasma. Le foie est rapidement prélevé et lavé dans du soluté physiologique $\left(4^{\circ} \mathrm{C}\right)$.

Les membranes plasmiques des foies groupés par lot sont préparées selon la méthode de Neville (1968), avec les modifications suivantes : les foies sont rapidement congelés dans l'azote liquide, conservés de 1 à 2 mois à $-80^{\circ} \mathrm{C}$ et décongelés en 2 à 5 min dans une solution de $\mathrm{NaHCO}_{3} 0,001 \mathrm{Mà} 4^{\circ} \mathrm{C}$. L'enrichissement en membranes des fractions est contrôlé par la mesure de l'activité enzymatique $5^{\prime}$-nucléotidase qui esł spécifiquement membranaire ; la contamination microsomiale est appréciée par l'activité enzymatique glucose-6-phosphatase. Les activités enzymatiques 5 -nucléotidase et glucose-6-phosphatase sont mesurées par les vitesses de libération de phosphore inorganique respectivement à partir d'adénosine-5'-monophosphate et de glucose-6phosphate (Aronson et Touster, 1974). Les protéines sont dosées selon la méthode de Lowry et al. (1951) ; l'albumine bovine (fraction V) est utilisée comme standard.

De l'insuline (Novo «monocomposée» lof $S$ 8381104) est iodée par 0,2 ou 0,5 atome d'iode (125l) par molécule, selon une méthode à la chloramine T ef purifiée sur colonne de DEAE cellulose (Freychet, 1974) ; cette technique permet d'obtenir principalement de la monoiodoinsuline (activité spécifique égale à 80 ou $180 \mu \mathrm{Ci} / \mu \mathrm{g}$ ) avec une activité biologique identique à celle de l'insuline standard (Freychet, Roth et Neville, 1971). La fixation spécifique de l'iodoinsuline aux membranes purifiées est étudiée selon les méthodes décrites par Freychet (1976b) (fig. 1) ; les incubations de $1 \mathrm{~h}$ a $30^{\circ} \mathrm{C}$ permettent d'obtenir la fixation à l'équilibre, chez des animaux au jeûne comme chez des animaux témoins (Broer, Freychet et Rosselin, 1977 ; Simon ef ol., 
1977). La capacité à fixer l'iodoinsuline et les constantes d'affinité des récepteurs d'insuline sont calculées au moyen de la représentation des résultats selon la méthode de Scatchard (fig. 2), analysée en fonction des deux événements suivants : - fixation de l'iodoinsuline à deux classes indépendantes de sites récepteurs (sites de haute affinité, basse capacité, et sites de faible affinité, haute capacité; Kahn, Freychet et Roth, 1974 ; tabl. 2) ; - fixation de l'iodoinsuline à une seule classe de sites récepteurs (récepteurs « vides》 ou « occupés») présentant une interaction entre les sites, de type coopérativité négative (De Meyts et Roth, 1975). La dégradation de l'iodoinsuline par les membranes, n'est pas mesurée. L'insuline plasmatique est dosée par radioimmunologie selon la technique du double anticorps au moyen des trousses in vitro CEA-IRE-SORIN INSIK-1; l'insuline standard est l'insuline humaine.

\section{Résultats et discussion.}

\section{Méthodologie}

L'activité 5'-nucléotidase (tabl. 3) est multipliée par environ 25 au cours du fractionnement du foie ; ceci traduit l'enrichissement important des fractions en membranes plasmiques. Elle est nettement plus faible chez les animaux soumis à la restriction énergétique que chez les animaux témoins ; elle semble aussi plus faible entre 2 et $4 \mathrm{~h}$ postprandiales que pendant les 2 premières heures postprandiales, excepté pour le lot $-E 1$; ces différences peuvent être dues aussi bien à des variations de l'activité spécifique 5'-nucléotidase dans la membrane qu'à des variations du rendement en protéines membranaires. L'activité glucose-6-phosphatase est plus faible dans les membranes purifiées que dans l'homogénat; ceci montre que la contamination des fractions membranaires par les microsomes est très faible. La quantité de protéines membranaires, corrigée pour le rendement de l'activité 5 '-nucléotidase, est plus forte chez les rats soumis à la restriction énergétique que chez les rats témoins, excepté pendant les 2 premières heures postprandiales pour le lot -E2.

La validité des mesures d'insuline est vérifiée. En effet, les dosages d'insuline réalisés dans les fractions obtenues à la suite du passage des plasmas groupés par lot à travers une colonne de séphadex G $50(0,9 \times 75 \mathrm{~cm})$, montre que l'immunoréactivité insulinique est pour environ 70 p. 100 associée à des substances de poids moléculaire identique à celui de l'insuline (tabl. 2). De plus, nous avons vérifié au moyen de la méthode des dilutions (Grizard et al., 1977), que les substances dosées dans un mélange de l'ensemble des plasmas, se fixent aux anticorps anti-insuline, sensiblement de la même manière que l'insuline standard humaine.

\section{Influence de la restriction énergétique}

La quantité d'énergie ingérée est environ 31 p. 100 plus faible chez les rats soumis à la restriction énergétique que chez les rats témoins, alors que la quantité de protéines ingérée est plus forte (15 p. 100) (łabl.1) ; la réduction de l'efficacité alimentaire apparente des protéines, beaucoup plus forte que celle de l'énergie, traduit l'accroissement de l'utilisation des protéines à des fins énergétiques (Grizard et al., 1975). La croissance des rats du lot témoin est plus faible que celle précédemment observée (Grizard et al., 1975) ; ceci est dû à l'ingestion plus faible à la suite du passage de l'alimentation ad libitum à l'alimentation par repas contrôlés, à un poids plus élevé. 
La restriction énergétique entraine une réduction non significative $(P \leqslant 0,050)$ de la teneur en insuline dans le plasma, associée à une augmentation non significative de la vitesse de renouvellement de cette hormone (tabl. 2). Ce résultał peuł être dû à une augmentation de la dégradation de l'insuline ou plus probablement à une diminution de la sécrétion à la suite de la réduction non significative de la glycémie (résultat non publié). Ce résultat est en accord avec les constatations selon lesquelles la diminution de la quantité d'aliment ingérée n'entraîne que peu de modifications de l'insulinémie, contrairement aux fortes baisses provoquées par le jeûne ou la réduction sévère de l'ingéré protéique (Trenkle, 1974 ; Atinmo ef al., 1976 ; Kabadi, Eisenstein et Strack, 1976 ; Edozien ef al., 1978). Quel que soit le régime consommé par les animaux, l'insulinémie est élevée pendant les 2 premières heures posłprandiales; ceci est dû aux

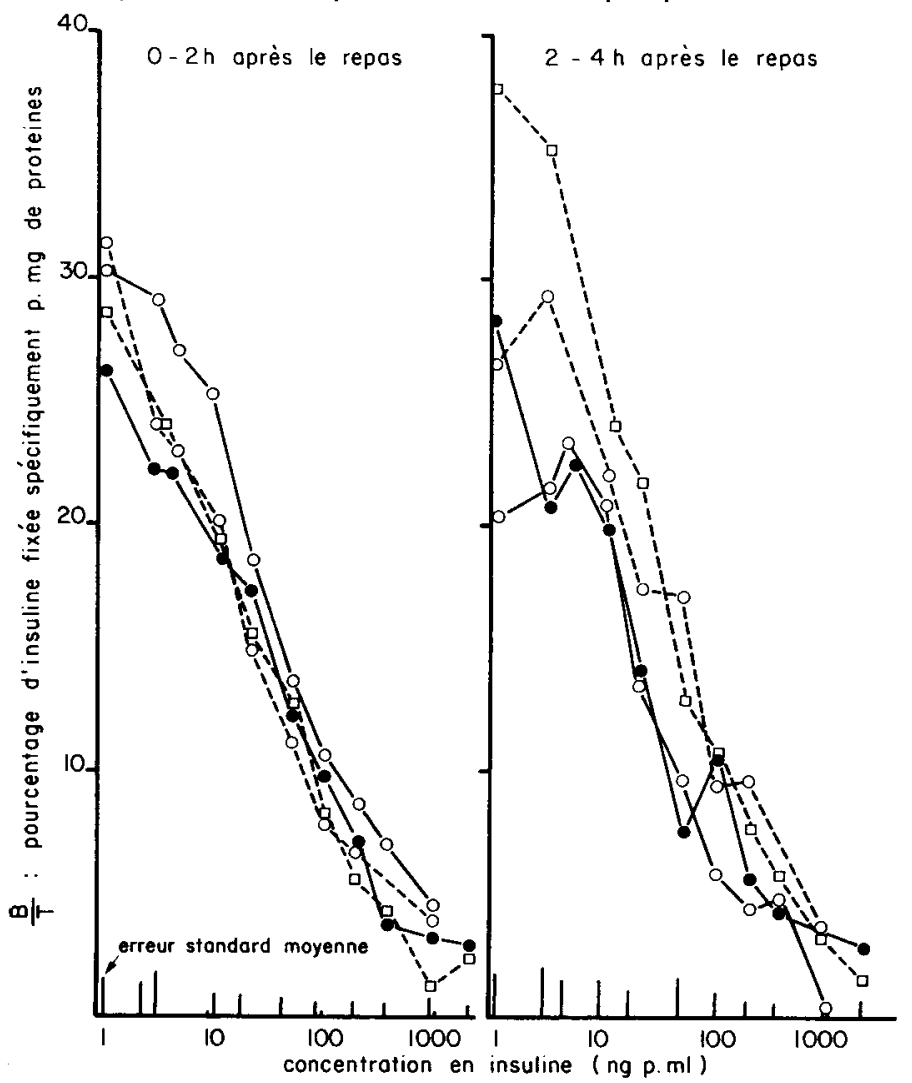

FIG. 1. - Fixation spécifique de l'iodoinsuline ${ }^{125}$ l aux membranes purifiées du foie en fonction de la concentration en insuline (-०-lot T ; - O- : lot -E1 ; - -O- - lot -E2 ; - - - - - lot -E3).

Les membranes purifiées $(0,15 \pm 0,02 \mathrm{mg}$ de protéines par $\mathrm{ml})$ sont incubées sous agitation $1 \mathrm{~h} \mathrm{à} 30^{\circ} \mathrm{C}$ dans un volume de $250 \mu \mathrm{l}$ de milieu Krebs Ringer Phosphate contenant de l'albumine bovine (14 $\mathrm{mg}$ par $\mathrm{ml}$ ) et l'iodoinsuline ${ }^{125}$ ( 1 ou $4 \mathrm{ng}$ par $\mathrm{ml}$ ) seule ou avec de l'insuline non radioactive ( 2 à $2000 \mathrm{ng}$ par $\mathrm{ml}$ ). Le pourcentage d'insuline fixée spécifiquement $(\mathrm{B} / \mathrm{T}$ ) est égal au pourcentage d'iodoinsuline fixée observé diminué du pourcentage d'insuline fixée non spécifiquement. Celui-ci est estimé par le pourcentage d'iodoinsuline fixée en présence d'une très forte concentration en insuline non radioactive $(50 \mu \mathrm{g}$ par $\mathrm{ml}$ ); il est égal à $11 \pm 2 \mathrm{p} .100$ par $\mathrm{mg}$ de protéines. Chaque point est la moyenne de 6 à 9 déterminations effectuées au cours de 2 à 3 expériences séparées. 
vée sous l'effet du jeûne (Broer, Freychet, et Rosselin, 1977 ; Almira et Reddy, 1979). Ce résultat n'est pas dû à l'accroissement de l'âge des animaux puisque la fixation de l'iodoinsuline aux membranes du foie du rat témoin, diminue légèrement de l'âge d'environ 50 jours à l'âge adulte (Freeman, Karoly ef Adelman, 1973 ; Kelly ef al., 1974).

Ensuite, entre 2 et $4 \mathrm{~h}$ après le repas, la fixation spécifique d'iodoinsuline ${ }^{125}$ aux membranes purifiées du foie des rats soumis à la restriction énergétique diminue et atteint des valeurs sensiblement identiques à celles observées chez les rats témoins (fig. 1). Cette baisse traduit soit une forte diminution de la concentration par $\mathrm{g}$ de foie en récepteurs de faible affinité (tabl. 2, fig. 2), soit une augmentation de la dégradation de l'iodoinsuline par les membranes. Cette baisse est en accord avec la réduction de la fixation spécifique d'iodoinsuline aux monocytes humains, observée après les repas (Beck-Nielsen et Pedersen, 1978 ; De Pirro ef al., 1979).

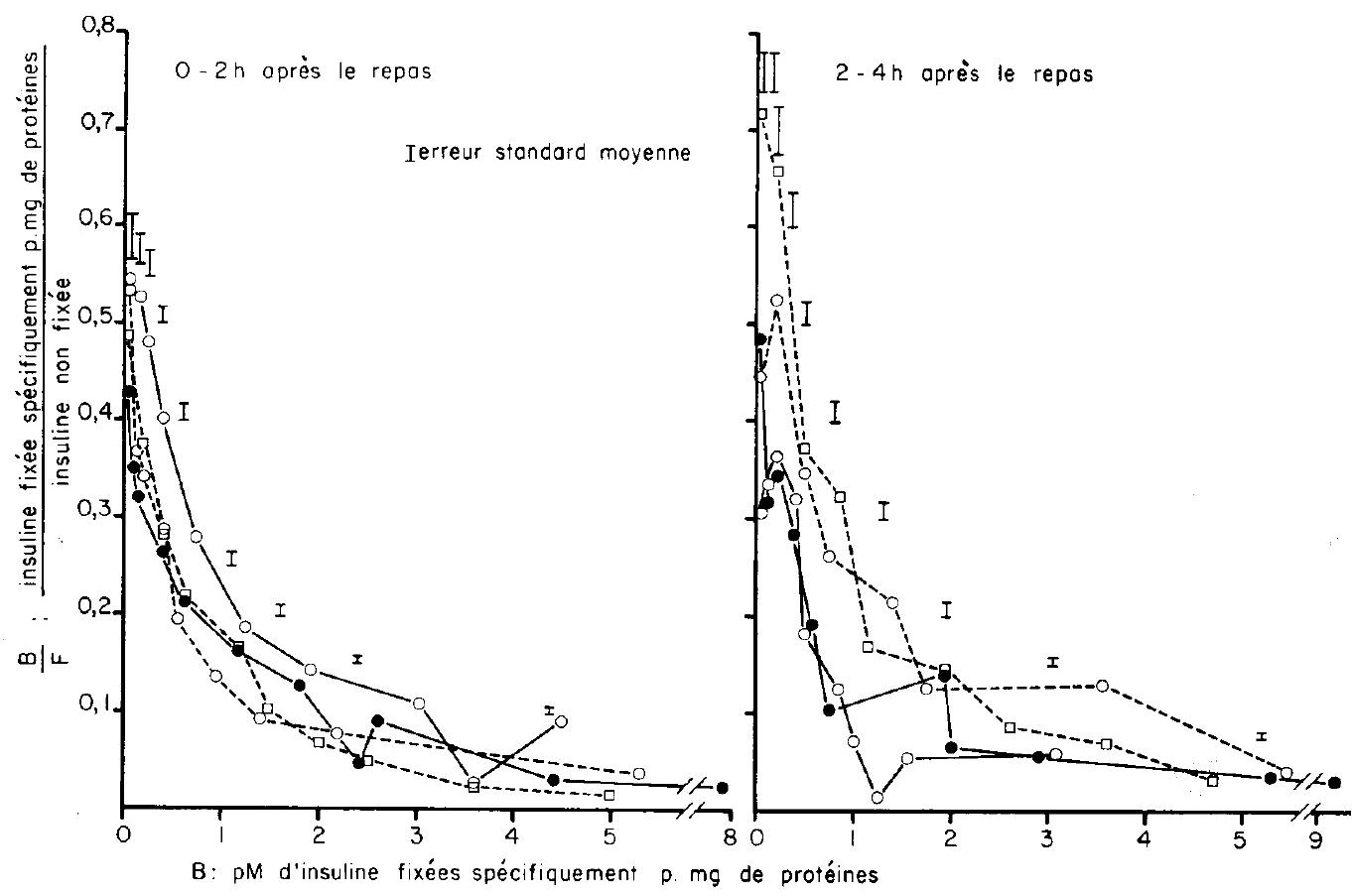

FIG. 2. - Présentation des résultats indiqués dans la figure 1 selon la méthode de Scatchard (- - : lot $\mathrm{T}$; -o- : lot -E1;--o-- lot -E2 ; --ם-- lot -E3). B : quantité d'insuline fixée spécifiquement = (pourcentage d'iodoinsuline fixée spécifiquement) $\times$ (concentration en insuline). B/F : quantité d'insuline fixée spécifiquement)/(quantité d'insuline non fixée) = (pourcentage d'iodoinsuline fixée spécifiquement $) /(100$ - pourcentage d'iodoinsuline fixée spécifiquement et non spécifiquement).

\section{Influence de l'hyperinsulinémie}

Les injections répétées d'insuline n'améliorent pas la croissance des animaux soumis à la restriction énergétique (tabl. 1), ni l'efficacité alimentaire apparente, contrairement à ce qui a été précédemment observé (Grizard ef al., 1975) ; cette contradiction peut être due aussi bien aux souches de rats différentes qu'aux traite- 
ments différents (nature et activité biologique de l'insuline injectée). Ce résultał s'explique en partie par une résistance aux effets de l'insuline pendant les 2 premières heures postprandiales (résultats non publiés) ; cela a d'ailleurs été observé chez les rałs qui reçoivent un régime équilibré ou un régime pauvre en protéines (Grizard, Prugnaud et Pion, 1977).

Les injections d'insuline provoquent de fortes augmentations de l'insulinémie (tabl. 2). Il en résulte que la vitesse de renouvellement de l'insuline est sensiblement diminuée chez les rats qui reçoivent une seule injection. En revanche, la vitesse de renouvellement de l'insuline est inchangée chez les rats qui reçoivent habituellement l'hormone ; ceci est dû au fait que la sécrétion d'insuline endogène est sans doute diminuée (Dunbar et al., 1976) ou le catabolisme de l'insuline accru.

Pendant les 2 premières heures après le repas, les injections d'insuline entraînent une baisse sensible de la fixation spécifique d'iodoinsuline ${ }^{125}$ aux membranes purifiées du foie, jusqu'à des valeurs légèrement supérieures à celles observées chez les rats témoins (fig. 1). Cetłe baisse traduit une diminution significative des constantes d'affinité (récepteurs «vides» et récepteurs « occupés») seulement chez les rats qui reçoivent une seule injection (tabl. 2, fig. 2). Cette baisse, constatée aussi bien in vivo qu'in vitro (Soll ef al., 1975 ; Olefsky et Reaven, 1975, Kahn ef al., 1977, Kobayashi et Olefsky, 1978), est due à une diminution de la synthèse ou plus vraisemblablement à une augmentation de la dégradation des récepteurs (Kahn et al., 1977) sans doute à la suite d'une élévation du passage dans le cytoplasme du complexe hormone-récepteur (Goldfine et al., 1977). Cette baisse ne semble pas résulter d'une inhibition de la fixation de l'iodoinsuline aux récepteurs par de l'insuline non radioactive fixée aux récepteurs avant le sacrifice des rats (Soll et al., 1975 ; Olefsky ef Reaven, 1975 ; Le MarchandBrustel, Jeanrenaud et Freychet, 1978).

La fixation spécifique d'iodoinsuline ${ }^{125}$ aux membranes purifiées du foie des rats traités par l'insuline, augmente fortement de la première à la deuxième période postprandiale étudiée ; chez les rałs qui reçoivent habituellement l'hormone, elle atteint des valeurs beaucoup plus fortes que dans tous les autres lots (fig. 1). Ceci traduit une augmentation de la concentration par $\mathrm{g}$ de foie en récepteurs de haute affinité chez tous les rats ; de plus, la concentration par $g$ de foie en récepteurs de faible affinité est augmentée chez les rats qui reçoivent une seule injection, ainsi que la constante d'affinité des récepteurs « vides» chez les rats qui reçoivent habituellement l'hormone (tabl. 2, fig. 2). Ceci est vraisemblablement dû à une augmentation de la synthèse ou une réduction du catabolisme des récepteurs, associée à la baisse de l'insulinémie. Ce résultat est en accord avec l'augmentation de la fixation spécifique de l'iodoinsuline aux adipocytes de rat, constatée par Kobayashi et Olefsky (1978), quelques heures après l'arrêt des injections répétées de fortes doses d'insuline. Ce résultat montre que la résistance des animaux aux effets de l'insuline n'est pas due à une baisse de la fixation spécifique d'iodoinsuline aux récepteurs d'insuline dans le foie, entre les repas de 14 et $18 \mathrm{~h}$.

\section{Conclusions.}

La restriction énergétique entraîne une augmentation de la fixation spécifique de l'iodoinsuline aux récepteurs d'insuline dans le foie. Celle-ci peut expliquer l'augmen- 
tation de la synthèse protéique hépatique constatée par Arnal, Fauconneau et Pech (1972). Les injections de fortes doses d'insuline induisent une résistance aux effets de l'insuline chez les rats soumis à la restriction énergétique comme chez les rats qui reçoivent un régime équilibré ou un régime pauvre en protéines (Grizard, Prugnaud ef Pion, 1977). Cette résistance à l'insuline n'est pas due à une réduction de l'activité des récepteurs d'insuline dans le foie.

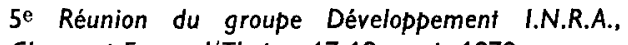
Clermont-Ferrand/Theix, 17-18 mai 1979.

\section{Références}

ARNAL M., FAUCONNEAU G., PECH R., 1972. Synthèses protéiques in vivo dans divers tissus du rat en croissance soumis à une réduction de l'apport énergétique de la ration. Ann. Biol. anim. Bioch. Biophys., 12, 91-108.

ALMIRA E. C., REDDY W. J., 1979. Effect of fasting on insulin binding to hepatocytes and liver plasma membranes from rats. Endocrinology, 104, 205-211.

ARONSON N. N., TOUSTER O., 1974. Isolation of rat liver plasma membrane fragments in isotonic sucrose, 90-102. In FLEISCHER S., PACKER L., Methods in Enzymology, vol. 31, part A, Acad. Press New York, San Francisco, London.

ATINMO T., BALDIJAO C., POND W. G., BARNES R. H., 1976. Plasma insulin levels in weaned pigs fed protein or energy restricted diets. J. Nutr., 106, 1654-1658.

BELLEMANN P., FRY J. R., BRIDGES J. W., SCHLOTE W., MECKE D., 1977. Effect of insulin on glycogen and protein synthesis in monolayer cultures of hepatocytes from normal and alloxan diabetic rats. Diabetologia, 13, 621-628.

BECK-NIELSEN H., PEDERSEN O., 1978. Diurnal variation in insulin binding to human monocytes. J. clin. Endocr. Mefab., 47, 385-390.

BROER Y., FREYCHET P., ROSSELIN G., 1977. Insulin and glucagon receptor interactions in genetically obese Zucker rat : studies of hormone binding and glucagon-stimulated cyclic AMP levels in isolated hepalocytes. Endocrinology, 101, 236-249.

De MEYTS P., ROTH J., 1975. Cooperativity in ligand binding : a new graphic analysis. Bioch. Biophys. Res. Com., 66, 1118-1126.

De PIRRO R., BERTOLI A., GRECO A. V., GELLI A. S., LAURO R., 1979. The effect of food intake on insulin receptor in man. Acta endocr., 90, 473-480.

DUNBAR J. C., MC LAUGHLIN W. J., WALSH M.-F. J., FOA P. P., 1976. Insulin secretion and glucose uptake by isolated islets of the hamster. Effect of insulin, proinsulin and C-peptide. Horm. Metab. Res., 8, 1-6.

EDOZIEN J. C., NIEHAUS N., MAR M. H., MAKOUI T., SWITZER B. R., 1978. Diet-hormone interrelationships in the rat. J. Nufr., 108, 1767-1776.

FREEMAN C., KAROLY K., ADELMAN R. C., 1973. Impairments of availability of insulin to liver in vivo and in binding of insulin to purified hepatic plasma membrane during aging. Biochem. Biophys. Res. Com., 54, 1573-1580.

FREYCHET P., 1974. The inferactions of proinsulin with insulin receptors on the plasma membrane of the liver. J. clin. Invest., 54, 1020-1031.

FREYCHET P., 1976a. Interactions of polypeptide hormones with cell membrane specific receptors: studies with insulin and glucagon. Diabetologia, 12, 83-100.

FREYCHET P., 1976b. Insulin receptors, 385-428. In BLECHER M., DEKKER M., New York. Methods in Receptor Research.

FREYCHET P., ROTH J., NEVILLE D. M., 1971. Monoiodoinsulin : demonstration of its biological activity and binding to fat cells and liver membranes. Biochem. Biophys. Res. Com., 43, 400-408.

FULKS R. M., LI J. B., GOLBERG A. L., 1975. Effect of insulin glucose and amino acids on protein turnover in rat diaphragm. J. biol. Chem., 250, 290-298.

GOLDFINE I. D., SMITH G. J., WONG K. Y., JONES A. L., 1977. Cellular uplake and nuclear binding of insulin in human cultured lymphocytes : evidence for potential intracellular sites of insulin action. Proc. nat. Acod. Sci. USA, 74, 1368-1372. 
GRIZARD J., PRUGNAUD J., ARNAL M., PION R., 1975. Effet de l'insuline sur la composition corporelle ef les teneurs en acides aminés libres du sang, du foie et du muscle du rat en croissance soumis d̀ une restriction énergétique. Ann. Biol. anim. Bioch. Biophys., 15, 569-582.

GRIZARD J., PRUGNAUD J., PION R., 1977. Influence d'un excès d'insuline sur la composition corporelle et les teneurs en acides aminés libres du sang, du foie et du muscle du rat en croissance soumis ou non à une restriction azotée. Ann. Biol. anim. Bioch. Biophys., 17, 373-387.

KABADI U. M., EISENSTEIN A. B., STRACK I., 1976. Decreased plasma insulin but normal glucagon in rats fed low protein diets. J. Nutr., 106, 1247-1253.

KAHN C. R., MEGYESI K., BAR R. S., EASTMAN R. C., FLIER J. S., 1977. Receptors for peptide hormones. New insights into the pathophysiology of disease states in man. Ann. int. Med., 86, 205219.

KAHN C. R., FREYCHET P., ROTH J., 1974. Quantitative aspects of the insulin-receptor interaction in liver plasma membranes. J. biol. Chem., 249, 2249-2257.

KANTER Y., 1976. The role of insulin in regulating protein catabolism in mammalian cells. Int. J. Biochem., 7, 253-257.

KELLY P. A., POSNER B. I., TSUSHIMA T., FRIESEN H. G., 1974. Studies of insulin, growth hormone and prolactin binding; ontogenesis, effects of sex and pregnancy. Endocrinology, 95, 532-539.

KOBAYASHI M., OLEFSKY J. M., 1978. Effect of experimental hyperinsulinemia on insulin binding and glucose transport in isolated rat adipocytes. Am. J. Physiol., 235, E53-E62.

LE MARCHAND-BRUSTEL Y., JEANRENAUD B., FREYCHET P., 1978. Insulin binding and effects in isolated soleus muscle of lean and obese mice. Am. J. Physiol., 234, E348-E358.

LOUIS-SYLVESTRE J., 1978. Relationship between two stages of prandial insulin release in rats. Am. J. Physiol., 235, E103-E111.

LOWRY O. H., ROSEBROUGH N. J., FARR A. L., RANDALL R. J., 1951. Protein measurement with the Folin phenol reagent. J. biol. Chem. 193, 265-275.

NEVILLE D. M., 1968. Isolation of an organ specific protein antigen from cell-surface membrane of rat liver. Biochim. biophys. Acta, 154, 540-552.

OLEFSKY J. M., REAVEN G. M., 1975. Effects of age and obesity on insulin binding to isolated adipocyles. Endocrinology, 96, 1486-1498.

PARILLA R., JIMENEZ M. I., AYUSO-PARILLA M. S., 1976. Cellular redistribution of metabolites during glucagon and insulin control of gluconeogenesis in the isolated perfused rat liver. Arch. Biochem. Biophys., 174, 1-12.

RÉRAT A., DESMOULIN B., 1970. Influence d'une restriction alimentaire, énergétique et azotée sur la croissance ef la composition corporelle du rat blanc. Ann. Zootech., 19, 103-115.

SIMON J., FREYCHET P., ROSSELIN G., DE MEYTS P., 1977. Enhanced binding affinity of chicken insulin in rat liver membranes and human lymphocytes : relationship to the kinetic properties of the hormone-receptor interaction. Endocrinology, 100, 115-121.

SOLL A. H., KAHN C. R., NEVILLE D. M., ROTH J., 1975. Insulin receptor deficiency in genetic and acquired obesity. J. clin. Invest., 50, 769-780.

TRENKLE A., 1974. Hormonal and nutritional interrelationships and their effects on skeletal muscle. J. anim. Sci., 38, 1142-1149. 